Article original

\title{
Recherche de variants génétiques de vulnérabilité à l'épilepsie chez des familles Algériennes
}

\author{
Characterization of genetic variants of vulnerability to epilepsy in Algerian families
}

Amina Chentouf', Mohand Laid Oubaiche', Malika Chaouch ${ }^{2}$

1Service de neurologie CHU d'Oran, Faculté de Médecine d'Oran, Algérie

2Faculté de Médecine d'Alger, Algérie

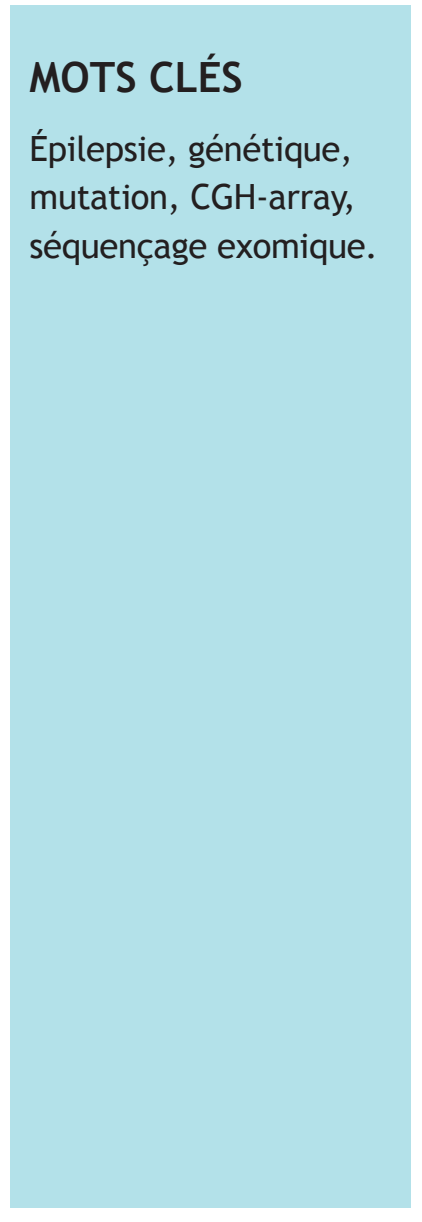

\section{Résumé}

Objectifs - Cette étude vise à caractériser des familles comptant plusieurs individus épileptiques, à étudier les modes de transmission de l'épilepsie au sein de ces familles, à rechercher des variants génétiques de vulnérabilité à l'épilepsie, et à analyser les relations génotype/phénotype.

Matériels et méthodes - Des familles multiplex ont été recrutées au service de neurologie du CHU d'Oran entre décembre 2011 et décembre 2016. Tous les participants ont été évalués cliniquement et ont bénéficié d'EEG et d'IRM cérébrales. Les syndromes épileptiques ont été classés selon les recommandations de la ligue internationale contre l'épilepsie (LICE) et les modes de transmission ont été déterminés à travers l'analyse généalogique. Après extraction de l'ADN génomique, des variants génétiques de susceptibilité à l'épilepsie ont été recherchés par hybridation génomique comparative sur micro-réseaux d'ADN (CGH-array) et par séquençage de nouvelle génération (NGS).

Résultats - Soixante cinq familles épileptiques ont participé à cette étude. L'âge moyen de début de la maladie était de $9.5 \pm 6.1$ ans avec une légère prédominance masculine (sex-ratio : 1.35). Les crises généralisées étaient légèrement plus fréquentes que les crises focales ( $50 \%$ vs. $40 \%$ ). Le taux de consanguinité parentale était de $50 \%$. Une concordance phénotypique a été constatée dans $2 / 3$ des familles. En tenant compte de l'analyse des pedigrees, l'épilepsie était transmise sur un mode autosomique dominant (AD)dans 29 familles (44.6\%) et sur un mode autosomique récessif (AR)dans 23 familles (35.4\%). Les analyses génétiques ont permis d'identifier des mutations du gène EPM1 chez des patients atteints d'épilepsie myoclonique progressive, une mutation du gène RELN chez des individus avec épilepsie du lobe temporal

Adresse de l'auteur correspondant : a.chentouf@facmed-univ-oran.dz 


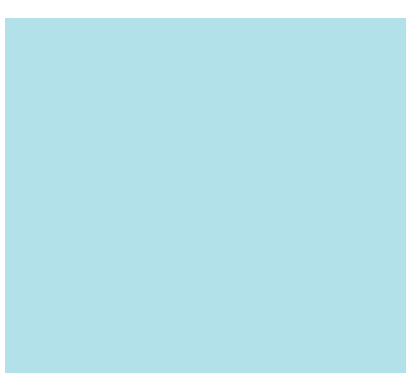

\section{KEY WORDS}

Epilepsy, genetics, mutation, CGH array, exome sequencing

\section{Introduction}

L'épilepsie est l'une des affections neurologiques les plus fréquentes, touchant près de 50 millions d'individus dans le monde dont $80 \%$ vivent dans des pays en voie de développement[1]. L'existence d'une composante génétique dans l'épilepsie est connue depuis des années grâce aux études épidémiologiques de concordance entre jumeaux et d'agrégation familiale, qui fournissent des preuves convaincantes pour l'héritabilité de cette maladie[2,3]. Par ailleurs, les études de liaison et les études d'associations pangénomiques ont permis l'isolement de gènes candidats intéressants [4-6].

\section{Abstract}

(ELT) et schizophrénie, ainsi que des variations du nombre de copies d'ADN (CNVs) bénignes et pathogènes. Par ailleurs,une mutation de novo (p.A39E) dans le gène GAL a été identifiée chez des jumeaux monozygotes atteints d'ELT, avec confirmation de l'implication du peptide muté dans le phénotype épileptique par des études in silico.

Conclusion - Cette étude a permis dedresser le phénotype et déterminer le mode de transmission de l'épilepsie chez des familles algériennes multiplex, et d'identifier des variants génétiques connus mais aussi des néomutations intéressantes.

Objectives - This study aims to investigate the surrounding family when several cases of epileptics are found, the goal is to establish inheritance patterns and to identify genetic variants and to document the genotype/phenotype correlation.

Materials and methods - Affected members from extended families with familial epilepsy were assessed at the University Hospital of Oran between December 2011 and December 2016. All participants underwent neurological examination, EEG and brain MRI. Epileptic syndromes have been classified according to the International League Against Epilepsy (LICE) criteria, and the modes of inheritance have been established through genealogical analysis. After genomic DNA extraction, genetic variants of susceptibility to epilepsy were investigated by comparative genomic hybridization on DNA microarrays (CGH-array) and next-generation sequencing (NGS).

Results - Sixty-five epileptic families participated in this study. The mean age of seizure onset was $9.5 \pm 6.1$ years with a slight male predominance (sex ratio: 1.35 ). 50\% had generalized seizures and $40 \%$ experienced focal seizures. The consanguinity rate among parents of affected was $50 \%$ with phenotypic concordance observed in $2 / 3$ of families. According to pedigree analysis, epilepsy was inherited in an AD mode with or without reduced penetrance in 29 families (44.6\%) and AR mode in 23 families (35.4\%). Genetic analyzes have identified mutations in the EPM1 gene in patients with progressive myoclonic epilepsy, a mutation in the RELN gene in individuals with temporal lobe epilepsy and schizophrenia, and both benign and pathogenic CNVs. In addition, a de novo mutation (p.A39E) in the GAL gene was identified in monozygotic twins with temporal lobe epilepsy. Functional analysis strongly supports GAL as the causal gene for the TLE in this family.

Conclusion - This study described the epileptic phenotype and determined the mode of transmission of epilepsy in large multigenerational Algerian families, and identified known genetic variants but also interesting neomutations.
En dépit des avancées majeures des techniques d'exploration pangénomique, et à l'ère du diagnostic de l'épilepsie par des applications disponibles sur Smartphones [7], et de son traitement personnalisé par les cellules pluripotentes et par thérapie génique $[8,9]$, force est de constater que la situation est loin d'être satisfaisante.En effet, la plupart des gènes découverts ont été associés aux épilepsies monogéniques qui ne représentent que 1 à $2 \%$ de toutes les épilepsies génétiques, et il reste encore beaucoup de formes familiales à hérédité complexe, pour lesquelles les gènes de susceptibilité demeurent inconnus[10]. 
Ceci nous a incités à mettre en place un programme de recherche visant à caractériser des familles multiplex, à étudier les modes de transmission de l'épilepsie au sein de ces familles, à rechercher des variants génétiques de vulnérabilité à l'épilepsie, et à analyser les relations génotype/ phénotype.

\section{Matériels et méthodes}

Il s'agit d'une étude prospective menée au service de neurologie du CHU d'Oran entre décembre 2011 et décembre 2016.

\section{Recrutement des familles et évaluation clinique}

Des familles comptant chacune au moins deux individus épileptiques ont étérecrutées par le biais de la consultation spécialisée et ont fait l'objet d'une évaluation clinique, d'enregistrements électroencéphalographiques (EEG) périodiques et d'imagerie cérébrale par résonance magnétique (IRM). Les apparentés sains, ont également été évaluéscliniquement et par des enregistrements EEG systématiques.

Le questionnaire utilisé est celui de l'Institut d'épidémiologie neurologique et de Neurologie Tropicale de Limoges, soutenu par la LICE[11]. Des arbres généalogiques d'au moins trois générations ont été élaborés par le logiciel « Genial Pedigree ».

La version française de la DIGS (Diagnostic Interview for GeneticStudies) et le questionnaire NDDI-E (The NeurologicalDisordersDepressionlnventory for epilepsy)ont été utilisés pour évaluer les patients ayant des troubles mentaux associés $[12,13]$.

\section{Classification des crises et des syndromes épileptiques} Nous avons collecté les données inhérentes aux manifestations critiques en interrogeant les patients et les témoins des crises. Nous nous sommes basés sur les antécédents des patients, leur statut neurologique, leur types de crises, leurs enregistrements EEG et leur imagerie cérébrale pour classer les syndromes épileptiques selon les recommandations de la LICE [14].

\section{Définitions}

- Le cas-index (probant) a été défini comme le premier membre de la famille à avoir franchi le seuil de notre consultation spécialisée.

- L'âge de début de la maladie a été défini comme l'âge où le patient ou sa famille ont constaté la survenue de la première crise.

- La consanguinité a été définie comme une relation maritale entre deux individus qui partagent le même ancêtre ; le degré de consanguinité étant déterminé par le nombre total de générations qui séparent deux individus de leur ancêtre commun [15].

- Nous avons conclu à une concordance phénotypique lorsque tous les membres affectés présentaient les mêmes caractéristiques cliniques (type de crises, âge de début des crises, réponse au traitement et résultats de l'EEG et de l’imagerie cérébrale).

- Les modes de transmission ont été déterminés à partir de l'analyse des pedigrees. Une transmission AD était suggéréelorsque les deux sexes étaient atteints de façon verticale sur plusieurs générations. Une transmission AR était déduite lorsque les individus atteints appartenaient à une génération issue de parents apparemment indemnes, apparentés ou originaires du même village. Un mode de transmission récessif lié à l'X était suspecté lorsque l'affection épargnait les sujets de sexe féminin et lorsqu'aucune transmission «male-to-male » n'était observée.

- L'épilepsie a été considérée comme pharmacorésistante en cas de: (a) échec de deux antiépileptiques appropriés, (b) survenue d'au moins une crise par mois durant une période $\geq 18$ mois, et (c) pas plus de 3 mois sans crises au cours de ces 18 mois [16].

\section{Analyses statistiques}

Les données collectées ont été codées et saisies sur logiciel SPSS version 21.0 pour Mac OS X. Nous avons procédé à une analyse descriptive des cas-index, faisant appel au calcul des moyennes et des écarts-types pour les variables quantitatives et des pourcentages pour les variables qualitatives.

\section{Analyses génétiques}

Nous avons effectué des prélèvements de sang veineux des patients et de leurs apparentés sains, et extrait l'ADN génomique. Cet ADN a été acheminé au laboratoire de médecine génétique et développement de l'université de Genève pour être analysé, à la recherche de variants génétiques par différentes techniques. Certaines familles ont fait l'objet d'une recherche de Copy Number Variants (CNV) ou variations du nombre de copies d'ADN par hybridation génomique comparative sur puce ADN (CGH-array), et d'autres ont été génotypées par séquençage à haut débit afin de détecter les polymorphismes nucléotidiques simples. Enfin, les familles au phénotype d'épilepsie myoclonique progressive ont été investiguées par PCR et Southern blot.

Afin d'interpréter l'avalanche de résultats générés par ces technologies de pointe, nous avons consulté régulièrement plusieurs bases de données de variations génétiques telles que OMIM, UCSC, Exac, DGV et Decipher. 


\section{Analyses fonctionnelles}

Des études in silico ont été réalisées au département de Neurochimie de l'Université de Stockholm en Suède, pour confirmer l'implication des mutations dans le phénotype épileptique. Il s'agit d'une approche basée sur des outils bioinformatiques permettant de prédire la fonction de la protéine codée par le gène muté.

\section{Consentement éclairé}

Le protocole de cette étude a été soumis à l'approbation par consentement écrit de tous les participants dont le formulaire a été rédigé en arabe et en français. Pour les mineurs et les sujets présentant des troubles du jugement, le consentement éclairé fut signé par le tuteur légal du sujet atteint, et ce, conformément au code de déontologie médicale [17].

\section{Résultats}

\section{Caractéristiques cliniques de la cohorte}

Soixante cinq familles multiplex ont participé à cette étude. Nous avons collecté les données relatives à 1238 individus parmi lesquels 19\% souffraient d'épilepsie dont deux paires de jumeaux monozygotes. Le nombre moyen d'individus atteints par famille était de 4.La notion de consanguinité parentale a été relevée chez la moitié de nos familles (33/65) dont 25 au premier degré et 8 au second degré.

Nous avons constaté une concordance phénotypique dans 45 familles alors que dans les vingt autres, il y avait une grande variabilité clinique intrafamiliale.

\section{Caractéristiques cliniques des 65 probants}

Nous avons noté une légère prédominance masculine avec un sex-ratio estimé à 1.35 .

L'âge variait entre 4 et 59 ans avec une moyenne de $27.7 \pm 13.2$ ans. L'âge de début de la maladie variait entre trois mois et 22 ans (âge moyen : $9.5 \pm 6.1$ ans). Les crises généralisées étaient légèrement plus fréquentes que les crises focales (50\% vs $40 \%$ ). La distribution des cas-index en fonction de la classification syndromique de la LICE est présentée dans la Figure 1.

35 patients (53.8\%) avaient une épilepsie contrôlée par monoou bithérapie. Quarante cinq (69.2\%) étaient libres de crises depuis au moins 3 mois. Les vingt autres probants (30.8\%) souffraient d'épilepsie pharmacorésistante. Après évaluation psychiatrique, deux probants ont été diagnostiqués avec déficience intellectuelle et $18(27.7 \%)$ avaient une comorbidité psychiatrique.

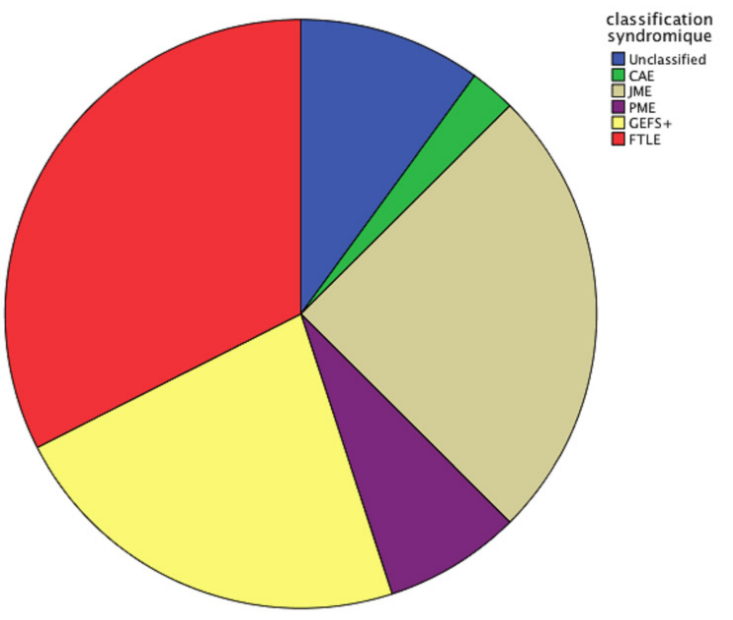

Figure 1: Distribution des probants en fonction de leur syndrome épileptique

CAE: épilepsie absence de l'enfant, JME: épilepsie myoclonique juvénile, EPM1: épilepsie myoclonique progressive de type 1, GEFS+: épilepsie génétique avec convulsions fébriles Plus, FTLE: épilepsie familiale du lobe temporal

\section{Modes de transmission}

En tenant compte de l'analyse des pedigree, l'épilepsie semble transmise sur un mode $A D$ avec ou sans pénétrance réduite dans 29 familles (44.6\%)et sur un mode AR dans 23 familles (35.4\%), alors qu'une transmission récessive liée à l' $X$ n'a été observée que dans une seule famille. Chez les 12 familles restantes, l'analyse généalogique n'était pas concluante ou suggérait plus d'un mode de transmission.

\section{Résultats des analyses génétiques et fonctionnelles}

A ce jour et pour des raisons financières, nous n'avons pu analyser génétiquement que 13 familles:

\subsection{Mutation du gène GAL et épilepsie du lobe temporal}

Cette famille originaire d'El Bayadh compte des jumeaux monozygotes atteints d'ELT évoluant depuis l'âge de 13 ans. Le séquençage exomique a permis d'identifier une mutation pA39E du gène GAL à l'état homozygote chez les jumeaux malades (Figure 2). Les analyses fonctionnelles ont révélé que le peptide muté p.A39E avait une activité antagoniste du récepteur GalR1, et une affinité réduite pour les récepteurs GalR2.

\subsection{Polymorphismes du gène RELN et vulnérabilité à l'épi- lepsie et à la schizophrénie}

Cette famille originaire d'Oran compte deux frères atteints d'ELT avec schizophrénie chez l'un, et trouble dépressif récurrent sur personnalité de type borderline chez l'autre. Le séquençage exonique a identifié un variant du gène RELN (rs55689103) à l'état homozygote chez l'individu avec schizophrénie, et à l'état hétérozygote chez son frère (Figure 3). 


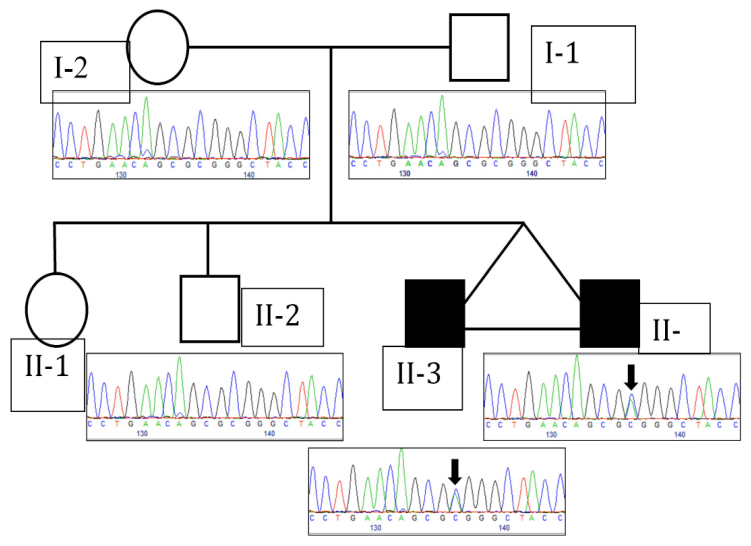

Figure 2: Résultats du séquençage exomique

\subsection{Mutations du gène EPM1 et épilepsie myoclonique pro- gressive type 1 (EMP1)}

Neuf familles au phénotype d’EMP1 ont été investiguées par PCR et Southern Blot.

Ces analysesont mis en évidence une expansion de plus de 30 copies du dodécamère CCCCGCCCCGCG dans le gène EPM1 chez tous les individus malades.

\subsection{Variations du nombre de copies d'ADN et épilepsie}

Une recherche de CNV par CGH array a été effectuée chez deux familles. La première comporte neuf individus épileptiques dont deux présentent en plus, une paraplégie spastique héréditaire complexe, et sept sont décédés d'une façon soudaine et inattendue (SUDEP). Dans cette famille, un seul CNV a été identifié en 7q21.12.

La deuxième famille comporte deux individus atteints d'épilepsie myoclonique juvénile, un individu avec mouvements anormaux et un autre avec déficience intellectuelle (DI) et

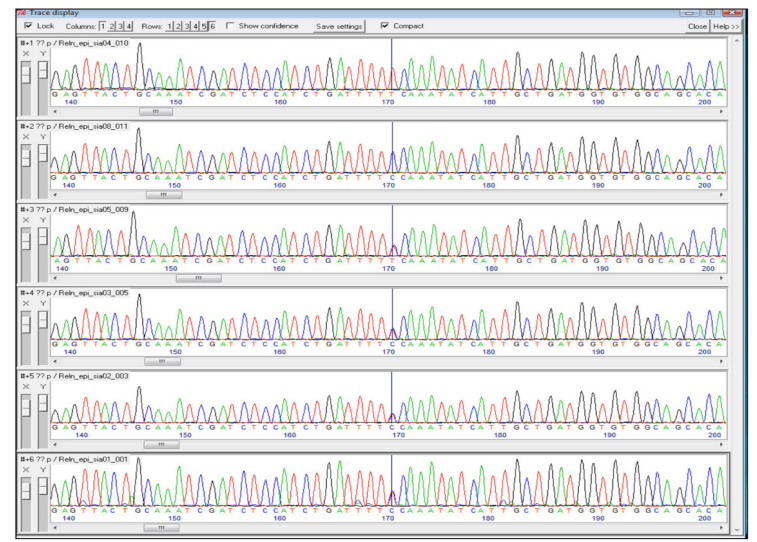

Figure 3 : Chromatographe de la séquence du gène RELN chez les membres de la famille syndrome dysmorphique. Dans cette famille, six CNV différents ont été identifiés dont un en 22q11.21 chez l'individu avec DI.

\section{Discussion}

\section{Caractéristiques cliniques de la cohorte}

Notre population d'étude est originaire d'Algérie, une région où le taux de mariages consanguins est estimé à $38 \%$. Dans notre cohorte, le taux de consanguinité estimé à 50\% était bien plus élevé que ce qui est attendu en population générale, suggérant un rôle de la consanguinité dans le déterminisme de certaines formes d'épilepsie génétique.En effet, dans une autre étude cas-témoin que nous avons menée au service de neurologie du CHU d'Oran, nous avons identifié la consanguinité comme facteur de risque significativement associé à l'épilepsie [18].

Nous avons relevé une grande variabilité clinique intrafamiliale dans $1 / 3$ des cas. Ce phénomène peut être expliqué par deux hypothèse:

- La première est celle du pléiotropisme génétique. En effet, certains gènes codent pour plusieurs traits phénotypiques à la fois. Par conséquent, une seule et même mutation peut s'exprimer par un large spectre phénotypique. C'est ce qu'on appelle « l'effet pléiotropique d'un gène ».

- La deuxième hypothèse qui a fait l'unanimité dans la communauté scientifique est celle de l'exposition à des facteurs épigénétiques qui vont moduler l'expression des gènes sans pour autant modifier la séquence d'ADN.

\section{Caractéristiques cliniques des 65 probants}

Nous avons relevé une légère prédominance masculine. La plupart des études épidémiologiques sur l'épilepsie montrent que les hommes sont plus fréquemment atteints que les femmes, cependant la différence est rarement statistiquement significative [19].

L'âge moyen de début des crises était de 9.5 ans, probablement en raison de la prédominance dans notre échantillon, de syndromes épileptiques qui ont la particularité de s'exprimer à l'adolescence ou un peu plus tard, tels que l'épilepsie myoclonique juvénile [20], l'épilepsie myoclonique progressive [21, 22] et l'épilepsie familiale du lobe temporal [23].

Les crises généralisées étaient légèrement plus fréquentes que les crises focales. Ce résultat est compatible avec des études menées dans des pays Arabes [24, 25], en Asie [26], en Afrique sub-Saharienne [27] et en Israël [28].

En effet, malgré l'existence d'une part génétique indéniable dans certaines formes familiales d'épilepsie focale, la contri- 
bution de ces facteurs reste relativement modeste par rapport à ce qui est rapporté dans les épilepsies généralisées [29].

Un tiers des proposants présentaient une épilepsie réfractaire. Ceci peut être en partie expliqué par la fréquence non négligeable de syndromes épileptiques myocloniques dans notre cohorte (EMJ ou EMP1), connus pour être pharmaco-résistants ou pharmacodépendants. En effet, dans une étude récente que nous avons menée au service de neurologie du CHU d'Oran, et qui avait pour objectif d'identifier les facteurs prédictifs précoces d'épilepsie réfractaire, les crises myocloniques étaient significativement prédictives de pharmaco-résistance [30]. Par ailleurs, la persistance des crises pourrait refléter une mauvaise observance thérapeutique de certains patients faisant face à des difficultés financières et à un niveau socio-économique très bas.

Des comorbidités psychiatriques étaient observées chez 18 probants, suggérant un lien entre l'épilepsie génétique et les troubles psychiatriques. En effet, il est actuellement admis que certaines affections psychiatriques telles que l'autisme , la schizophrénie et le trouble du déficit de l'attention avec ou sans hyperactivité, partagent des variants génétiques communs avec l'épilepsie [31].

\section{Modes de transmission}

Une transmission $A D$ avec ou sans pénétrance réduite a été constatée dans $45 \%$ des familles présentant des phénotypes d'ELT avec hallucinations auditives et d'épilepsie génétique avec convulsions fébriles Plus (GEFS+), connus pour leur transmission exclusivement AD. Une TAR était constatée dans $35 \%$ des cas chez des familles possédant plusieurs boucles de consanguinité, suggérant un effet fondateur commun.

\section{Analyses génétiques et fonctionnelles}

\subsection{Mutation du gène GAL et épilepsie du lobe temporal}

Le gène GAL est localisé en 11q13.2. Il code pour le neuropeptide Galanin qui possède des propriétés anticonvulsivantes reconnues depuis les années nonante, mais uniquement via des modèles animaux[32, 33]. A ce jour, aucune mutation pathogène du gène galanine n'avait encore été identifiée chez l'Homme.

Afind'analyser la relation génotype-phénotype, et de vérifier le lien de causalité entre la mutation pA39E identifiée chez nos jumeaux et leur phénotype épileptique, nous avons effectué des études in silico. Ces études ont mis en évidence une diminution de l'affinité du peptide muté pour les récepteurs de la galanine de type 2 et des propriétés antagonistes carrément proconvulsivantes, médiées par les récepteurs de type 1. Ainsi, nos résultats génétiques et fonctionnels sont en faveur d'un effet dominant négatif d'haplo-insuffisance comme mécanisme potentiel de causalité, suggérant que le variant p.A39E du gène GAL pourrait être impliqué dans la survenue d'une ELT. Nos résultats ont été répertoriés dans la base de données OMIM (Online Mendelianlnheritance in Man :https:// www.omim.org/entry/616461).

Ces résultats pourraient avoir des retombées directes sur le développement de nouveaux traitements antiépileptiques. En effet, les propriétés anticonvulsivantes de la galanine ont séduit l'industrie du génie génétique, et ont incité le développement de cellules encapsulées produisant ce neuropeptide [34-36]. Ces approches innovantes sont encore à l'état expérimental.

\subsection{Polymorphismes du gène RELN et vulnérabilité à l'épi- lepsie et à la schizophrénie}

Dans cette famille, le séquençage exonique a identifié un variant du gène RELN (rs55689103) à l'état homozygote chez l'individu avec ELT et schizophrénie, et à l'état hétérozygote chez son frère au phénotype d'ELT et dépression. Le gène RELN ([OMIM] 600514) est situé en 7q22.1. Il code pour la Reelin, une gigantesque protéine extracellulaire de 3460 acides aminés qui joue un rôle primordial dans le développement neuronal, dans la croissance dendritique, dans la plasticité synaptique nécessaire aux fonctions d'apprentissage et de mémoire, et dans la régulation des récepteurs N-Methyl-D-Aspartate (NMDA)[37, 38]. Les troubles du métabolisme de la Reelin se traduisent par un défaut de lamination corticale [39] ou une lissencéphalie [40, 41]. Ainsi, les mutations RELN pourraient être impliquées dans la pathogénèse de troubles neuropsychiatriques tels que la maladie d'Alzheimer, la démence fronto-temporale, les troubles du spectre autistique, les troubles bipolaires, certains syndromes épileptiques et la schizophrénie [42-45].

\subsection{Mutations du gène EPM1 et épilepsie myoclonique pro- gressive type 1 (EMP1)}

Chez les neuf cas index testés par Southern Blot, une expansion de plus de 30 copies du dodécamère CCCCGCCCCGCG dans le gène EPM1 a été identifiée à l'état homozygote. Ces résultats sont compatibles avec le diagnostic génétique d'épilepsie myoclonique progressive de type 1[46]. En effet, l'EMP1 est liée à des mutations du gène de la cystatine B (CSTB : NM_000100) cartographié sur le chromosome 21q22.3 chez des patients d'origine méditerranéenne, française et maghrébine [47, 48].

\subsection{Variations du nombre de copies d'ADN et épilepsie}

Dans la première famille, un seul CNV a été identifié en 7q21.12 chez la mère de la probante. Etant donné que la probante n'a pas hérité de ce CNV, son implication dans le phénotype de cette famille est très peu probable. Dans laseconde famille, six CNV différents ont été identifiés dont un en 22q11.21 chez 
l'individu avec déficience intellectuelle mais sans épilepsie. Du fait de la richesse de la région chromosomique 22q11.21 en gènes, les CNV de cette région sont responsables d'un large spectre phénotypique avec une grande variabilité inter et intrafamiliale, pouvant aller du retard mental léger au syndrome de DiGeorge [49, 50]. Ce CNV pourrait donc être responsable du phénotype de ce patient.

\section{Limites de l'étude}

Les limites de cette étude doivent être soulignées. D’abord, les difficultés d'ordre financier ; ces techniques coutent excessivement cher d'où le nombre restreint de familles analysées génétiquement. Ces techniques génèrent un déluge de résultats qui nécessite des outils bioinformatiques de pointe pour leur interprétation.

Une autre difficulté réside dans le fait que le génome nord-africain est peu représenté dans les bases de données internationales, d'où la difficulté de comparer nos résultats.

\section{Conclusion}

A travers cette étude, nous avons pu dresser le phénotype et déterminer le mode de transmission de l'épilepsie chez des familles algériennes multiplex, confirmant ainsi le caractère héréditaire de cette maladie. Nous avons identifié des variants génétiques connus mais aussi des néomutationsintéressantes pouvant avoir des retombées sur le développement de nouveaux traitements antiépileptiques.

\section{Conflits d'intérêts}

Les auteurs déclarent n'avoir aucun conflit d'intérêt.

\section{Remerciements}

Nous tenons à remercier les patients et leurs familles pour avoir accepté de participer à cette étude.La réalisation d'une partie des travaux présentés dans ce document a été rendue possible grâce au soutien financier obtenu auprès du Fonds National Suisse de la Recherche Scientifique (SNF-144082), du Centre de Compétences en Recherche Synapsy, du téléthon d'Italie (Grant no. GGP12078), de la Commission de Génétique de la Ligue Italienne contre l'épilepsie et du Conseil Suédois de la Recherche.

\section{Références bibliographiques}

1. WHO., Atlas: epilepsy care in the world. WHO press, 2005: p. 11-3.

2. Kjeldsen, M.J., L.A. Corey, K. Christensen, and M.L. Friis, Epileptic seizures and syndromes in twins: the importance of genetic factors. Epilepsy Res, 2003. 55(1-2): p. 137-46.

3. Ottman, R. and N. Risch, Genetic Epidemiology and Gene Discovery in Epilepsy, in Jasper's Basic Mechanisms of the Epilepsies, J.L. Noebels, et al., Editors. 2012: Bethesda (MD).

4. Brunklaus, A., R. Ellis, H. Stewart et al. Homozygous mutations in the SCN1A gene associated with genetic epilepsy with febrile seizures plus and Dravet syndrome in 2 families. Eur J PaediatrNeurol, 2015. 19(4): p. 484-8.

5. Buono, R.J., Genome wide association studies (GWAS) and common forms of human epilepsy. Epilepsy Behav, 2013. 28Suppl 1: p. S63-5.

6. International League Against Epilepsy Consortium on Complex Epilepsies. Electronic address, e.-a.u.e.a., Genetic determinants of common epilepsies: a meta-analysis of genome-wide association studies. Lancet Neurol, 2014. 13(9): p. 893-903.

7. Liu, X., R. Wang, D. Zhou et al. Feasibility and acceptability of smartphone applications for seizure self-management in China: Questionnaire study among people with epilepsy. Epilepsy Behav, 2016. 55: p. 57-61.

8. Jin, Y., C. Zhao, L. Chen et al. Identification of novel gene and pathway targets for human epilepsy treatment. Biol Res, 2016. 49(1): p. 3.

9. Shetty, A.K. and D. Upadhya, GABA-ergic cell therapy for epilepsy: Advances, limitations and challenges. NeurosciBiobehavRev, 2016. 62: p. 35-47.

10. Hardies, K., S. Weckhuysen, P. De Jonghe et al.Lessons learned from gene identification studies in Mendelian epilepsy disorders. Eur J Hum Genet, 2015.

11. Preux, P.M., [Questionnaire in a study of epilepsy in tropical countries]. Bull SocPatholExot, 2000. 93(4): p. 276-8.

12. Preisig, M., B.T. Fenton, M.L. Matthey, et al. Diagnostic interview for genetic studies (DIGS): inter-rater and test-retest reliability of the French version. Eur Arch Psychiatry ClinNeurosci, 1999. 249(4): p. 1749.

13. Alkhamees, H.A., C.E. Selai, S.D. Shorvon et al. The use of the NDDI-E in Arabic to identify symptoms of depression of moderate or greater severity in people with epilepsy. Epilepsy Behav, 2014. 32: p. 55-8.

14. Berg, A.T., S.F. Berkovic, M.J. Brodie et al. Revised terminology and concepts for organization of seizures and epilepsies: report of the ILAE Commission on Classification and Terminology, 2005-2009. Epilepsia, 2010. 51(4): p. 676-85.

15. Tadmouri, G.O., P. Nair, T. Obeid et al. Consanguinity and reproductive health among Arabs. Reprod Health, 2009. 6: p. 17.

16. Tellez-Zenteno, J.F., L. Hernandez-Ronquillo, S. Buckley et al. A validation of the new definition of drug-resistant epilepsy by the In- 
ternational League Against Epilepsy. Epilepsia, 2014. 55(6): p. 829-34.

17. Officiel, J., Règles de déontologie médicale. 1992: Algérie.

18. Chentouf, A., R. Talhi, A. Dahdouh et al.Consanguinity and epilepsy in Oran, Algeria: A case-control study. Epilepsy Res, 2015. 111: p. 10-7.

19. Hauser, W.A., J.F. Annegers, and W.A. Rocca, Descriptive epidemiology of epilepsy: contributions of population-based studies from Rochester, Minnesota. Mayo ClinProc, 1996. 71(6): p. 576-86.

20. Cvetkovska, E., S. Panov, and I. Kuzmanovski, Clinical genetic study in juvenile myoclonic epilepsy. Seizure, 2014. 23(10): p. 903-5.

21. Magaudda, A., E. Ferlazzo, V.H. Nguyen et al.Unverricht-Lundborg disease, a condition with self-limited progression: long-term follow-up of 20 patients. Epilepsia, 2006. 47(5): p. 860-6.

22. Shahwan, A., M. Farrell, and N. Delanty, Progressive myoclonic epilepsies: a review of genetic and therapeutic aspects. Lancet Neurol, 2005. 4(4): p. 239-48.

23. Aguglia, U., A. Gambardella, E. Le Piane, D et al. Mild non-lesional temporal lobe epilepsy. A common, unrecognized disorder with onset in adulthood. Can J Neurol Sci, 1998. 25(4): p. 282-6.

24. Moualek, D., L.A. Pacha, S. Abrouk et al. Multicenter transversal two-phase study to determine a national prevalence of epilepsy in Algeria. Neuroepidemiology, 2012. 39(2): p. 131-4.

25. Hamdy, N.A., Prevalence of epilepsy in primary school children in Elminia city, Egypt. Egypt J. Neurol. Psychiat. Neurosurg., 2009. 46(1): p. 33-39.

26. Mac, T.L., D.S. Tran, F. Quet et al. Epidemiology, aetiology, and clinical management of epilepsy in Asia: a systematic review. Lancet Neurol, 2007. 6(6): p. 533-43.

27. Preux, P.M. and M. Druet-Cabanac, Epidemiology and aetiology of epilepsy in sub-Saharan Africa. Lancet Neurol, 2005. 4(1): p. 21-31.

28. Afawi, Z., K.L. Oliver, S. Kivity et al. Multiplex families with epilepsy: Success of clinical and molecular genetic characterization. Neurology, 2016. 86(8): p. 713-22.

29. Corey, L.A., J.M. Pellock, M.J. Kjeldsen et al. Importance of genetic factors in the occurrence of epilepsy syndrome type: a twin study. Epilepsy Res, 2011. 97(1-2): p. 103-11.

30. Chentouf, A., A. Dahdouh, S. Ghomari et al. Early predictors of intractable epilepsy in Oran, Algeria: A case-control study. International Journal of Neurology and Brain Disorders, 2016. 3(2): p. 1-5.

31. Chentouf, A., Epilepsy and Psychiatric Disorders: Is There a Common Genetic Susceptibility? Epilepsy Journal, 2016. 2(2).

32. Mazarati, A.M., E. Halaszi, and G. Telegdy, Anticonvulsive effects of galanin administered into the central nervous system upon the picrotoxin-kindled seizure syndrome in rats. Brain Res, 1992. 589(1): p. 164-6.

33. Mazarati, A., X. Lu, K. Kilk et al. Galanin type 2 receptors regulate neuronal survival, susceptibility to seizures and seizure-induced neuro- genesis in the dentate gyrus. Eur J Neurosci, 2004. 19(12): p. 3235-44.

34. Kanter-Schlifke, I., A. Toft Sorensen, M. Ledri et al. Galanin gene transfer curtails generalized seizures in kindled rats without altering hippocampal synaptic plasticity. Neuroscience, 2007. 150(4): p. 984-92.

35. Lu, X., E. Roberts, F. Xia et al.GalR2-positive allosteric modulator exhibits anticonvulsant effects in animal models. Proc Natl Acad Sci U S A, 2010. 107(34): p. 15229-34.

36. Nikitidou, L., M. Torp, L. Fjord-Larsen et al. Encapsulated galanin-producing cells attenuate focal epileptic seizures in the hippocampus. Epilepsia, 2014. 55(1): p. 167-74.

37. Finkbeiner, S., S.F. Tavazoie, A. Maloratsky et al. CREB: a major mediator of neuronal neurotrophin responses. Neuron, 1997. 19(5): p. 1031-47.

38. Teixeira, C.M., M.M. Kron, N. Masachs et al.Cell-autonomous inactivation of the reelin pathway impairs adult neurogenesis in the hippocampus. J Neurosci, 2012. 32(35): p. 12051-65.

39. D'Arcangelo, G., G.G. Miao, S.C. Chen et al.A protein related to extracellular matrix proteins deleted in the mouse mutant reeler. Nature, 1995. 374(6524): p. 719-23.

40. Fatemi, S.H., Reelin mutations in mouse and man: from reeler mouse to schizophrenia, mood disorders, autism and lissencephaly. Mol Psychiatry, 2001. 6(2): p. 129-33.

41. Willemsen, M.H., L.E. Vissers, and M. Willemsen, Mutations in DY$\mathrm{NC} 1 \mathrm{H} 1$ cause severe intellectual disability with neuronal migration defects. J Med Genet, 2012. 49: p. 179-183.

42. Fatemi, S.H., A.V. Snow, J.M. Stary et al. Reelin signaling is impaired in autism. Biol Psychiatry, 2005. 57(7): p. 777-87.

43. Liu, J.S., Molecular genetics of neuronal migration disorders. Curr Neurol Neurosci Rep, 2011. 11(2): p. 171-8.

44. Fatemi, S.H., J.L. Kroll, and J.M. Stary, Altered levels of Reelin and its isoforms in schizophrenia and mood disorders. Neuroreport, 2001. 12(15): p. 3209-15.

45. Imai, H., H. Shoji, M. Ogata et al.Dorsal Forebrain-Specific Deficiency of Reelin-Dab1 Signal Causes Behavioral Abnormalities Related to Psychiatric Disorders. Cereb Cortex, 2016.

46. Lalioti, M.D., M. Mirotsou, C. Buresi et al. Identification of mutations in cystatin $B$, the gene responsible for the Unverricht-Lundborg type of progressive myoclonus epilepsy (EPM1). Am J Hum Genet, 1997. 60(2): p. 342-51.

47. Malafosse, A., A.E. Lehesjoki, P. Genton et al.Identical genetic locus for Baltic and Mediterranean myoclonus. Lancet, 1992. 339(8801): p. 1080-1.

48. Joensuu, T., A.E. Lehesjoki, and O. Kopra, Molecular background of EPM1-Unverricht-Lundborg disease. Epilepsia, 2008. 49(4): p. 557-63.

49. Kim, E.H., M.S. Yum, B.H. Lee et al. Epilepsy and Other Neuropsychiatric Manifestations in Children and Adolescents with 22q11.2 Deletion Syndrome. J Clin Neurol, 2016. 12(1): p. 85-92. 
50. Vaz, S.O., R. Pires, L.M. Pires et al. A unique phenotype in a patient with a rare triplication of the 22q11.2 region and new clinical insights of the 22q11.2 microduplication syndrome: a report of two cases. BMC Pediatr, 2015. 15: p. 95. 
